\title{
Spin coupling around a carbon atom vacancy in graphene
}

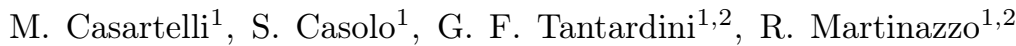 \\ ${ }^{1}$ Dipartimento di Chimica, Università degli Studi di Milano, via Golgi 19, 20133 Milan, Italy and \\ ${ }^{2}$ Istituto di Scienze e Tecnologie Molecolari, CNR, via Golgi 19, 20133 Milan, Italy
}

\begin{abstract}
We investigate the details of the electronic structure in the neighborhoods of a carbon atom vacancy in graphene by employing magnetization-constrained density-functional theory on periodic slabs, and spin-exact, multi-reference, second-order perturbation theory on a finite cluster. The picture that emerges is that of two local magnetic moments (one $\pi$-like and one $\sigma$-like) decoupled from the $\pi$ band and coupled to each other. We find that the ground state is a triplet with a planar equilibrium geometry where an apical $\mathrm{C}$ atom opposes a pentagonal ring. This state lies $\sim 0.2 \mathrm{eV}$ lower in energy than the open-shell singlet with one spin flipped, which is a bistable system with two equivalent equilibrium lattice configurations (for the apical $\mathrm{C}$ atom above or below the lattice plane) and a barrier $\sim 0.1 \mathrm{eV}$ high separating them. Accordingly, a bare carbon-atom vacancy is predicted to be a spin-one paramagnetic species, but spin-half paramagnetism can be accommodated if binding to foreign species, ripples, coupling to a substrate, or doping are taken into account.
\end{abstract}

\section{INTRODUCTION}

Magnetism in graphene is a fascinating and highly controversial matter ${ }^{1}$. Early reports on ferromagnetic ordering in graphite and graphene ${ }^{2 / 5}$ have been questioned in the light of the ubiquitous presence of magnetic contaminants, and measurements under carefully controlled conditions showed that graphene, like graphite, is strongly diamagnetic with a weak paramagnetic contribution from adatoms and/or carbon atom vacancies6. Simple adsorbates such as fluorine and missing carbon atoms have been shown to provide a spin- $1 / 2$ paramagnetic response ${ }^{7}$, though spin-1 paramagnetism has been reported upon $\mathrm{N}^{+}$ irradiation 8 . Direct evidences of the presence of adatom- and vacancy-related magnetic moments have been observed in pure spin transport measurements on graphene spin valves 9 . Likewise, signatures of Kondo effect have been observed in charge-transport measurements at low temperatures on irradiated graphene, and high Kondo temperatures reported for finite and zero electron density $\sqrt{10}$, at odds with the above magnetometry measurements 7 .

From a theoretical perspective, perfect bipartite systems support a number of zero-energy "midgap" states which is greater or equal than the sublattice imbalance $\left|n_{A}-n_{B}\right|$, where $n_{A}, n_{B}$ are the number of sites in the two sublattices 1112. When imbalance results from isolated missing $p_{z}$ orbitals (e.g. for low concentrations of covalently bound adatoms or vacancies) these states decay slowly $(\sim 1 / r)$ from the defects and localize on the locally majority sites ${ }^{13 / 14}$, as confirmed by experiments 15 . Thus, these defects form quasi-localized $\pi$ moments, which couple either ferromagnetically or antiferromagnetically depending on their lattice position. In fact, with local interactions only, at charge neutrality (half-filling) the spin state of the system exactly matches the sublattice imbalance ${ }^{16}, S=\left|n_{A}-n_{B}\right| / 2$, and thus coupling is ferromagnetic for defects in the same sublattice and antiferromagnetic otherwise, a result which also fol- lows by analyzing RRKY interactions in graphene $e^{17}$. Within the same assumptions (perfect electron-hole symmetry, local interactions only) coupling between $\pi$ moments and conduction states has been investigated beyond mean-field approaches and found to be ferromagnetic $\frac{18}{}$, thereby confirming that simple adatoms covalently bound to the substrate (e.g. $\mathrm{H}$, $\mathrm{F}$ species) behave as spin-1/2 localized moments. In turn, this also affects chemical properties and favors formation of dimers of balanced type $e^{19120}$.

This simple picture has to be revised for a carbon atom vacancy where, in addition to the above $\pi$ midgap state, three $\sigma$ orbitals are left singly occupied upon vacancy formation, and a structural instability (Jahn-Teller distortion) arises which breaks electron-hole symmetry, even if nearest neighbors interactions only are retained. The ensuing lattice re-arrangement leaves two unpaired electrons, and a magnetic moment in the range $2.0-1.0 \mu_{B}$ has been found by (ensemble) density functional theory (DFT) calculations 21 26, with a clear tendency to $1.0 \mu_{B}$ (and vanishing dependence of the energy on the magnetization) in the low-density limit which signals the absence of any order at experimentally relevant concentrations 26! Apart from the possible role of electron-hole symmetry, this apparently contrasts with the situation described above for a $\pi$ moment only, since the presence of an additional unpaired electron in a very localized $\sigma$ orbital is expected to give either a triplet or a singlet state. Recent experiments have shown that the spin $-1 / 2$ paramagnetism of missing carbon atoms has two contributions 27 , from $\sigma$ and $\pi$ states respectively, and one of them can be quenched upon molecular doping and possibly by means of the electric field effect ${ }^{27}$. This result requires that, at least under the experimental conditions of Ref. 27, the unpaired electrons around a vacancy negligibly interact with each other, in contrast with early reports on spin-1 paramagnetism of irradiated graphene samples 8 . Proper consideration of $\sigma$ states, and their possible hybridization with $\pi$ states when 
the substrate is no longer locally planar, e.g. because of ripples or interaction with a substrate 28 , has led to reconsidering the issue of the interaction between the localized magnetic moments and the conduction electrons ${ }^{29 \mid 30}$, though the most recent experiments 27 seem to rule out this possibility.

Here, in order to help shed light on the above issues we consider in detail the electronic structure around a carbon atom vacancy in graphene. We employ both conventional DFT methods in periodic models and accurate, spin-exact quantum chemistry methods in a finite cluster, to investigate the coupling of the two electrons left unpaired after vacancy formation and reconstruction. We analyze several substrate geometries close to the equilibrium one, and focus in particular on the out-of-plane movement of the carbon atom where most of the unpaired electron density resides. Our results show that the triplet is the ground-state and has a planar equilibrium geometry, while the singlet -which lies $\sim 0.2 \mathrm{eV}$ above it - gets easily stabilized by an out-of-plane lattice distortion. Hence, both spin-1 and spin-1/2 paramegnetism may in principle arise in irradiated graphene, depending on local interactions, curvature, etc. of the graphene sheet, in addition to doping or chemical interactions with foreign species.

The manuscript is organized as follows: Section II outlines the important Jahn-Teller distortion occurring in the system and Section III gives the details of the electronic structure methods adopted in this work. Results are given in Section IV and discussed in Section V, and Section VI summarizes and concludes.

\section{JAHN-TELLER DISTORTION}

As mentioned above, formation of a carbon atom vacancy gives rise to localized states around the vacancy, namely one $\pi$ (semilocalized) "midgap" state and three dangling orbitals in the $\sigma$ network which result from breaking the $s p^{2}$ bonds which hold the carbon atom in place. In the local $D_{3 h}$ point symmetry group which is appropriate to discuss proper and pseudo Jahn-Teller distortions, the first belongs to $a_{2}^{\prime \prime}$ symmetry species, and the latter span $a_{1}^{\prime}+e^{\prime}$ irreducible representations, $a_{1}^{\prime}$ being lowest in energy since it contains a purely bonding combination of $\sigma$ orbitals. Hence, the lowest energy "scenarios" for the many-body electronic state can be obtained by distributing two electrons in the $e^{\prime}$ and $a_{2}^{\prime \prime}$ states, i.e. starting from configurations of the type .. $\left(a_{1}^{\prime}\right)^{2}\left(e^{\prime}\right)^{n_{1}}\left(a_{2}^{\prime \prime}\right)^{n_{2}}$ with $n_{1}+n_{2}=2$. Among these, the one with $n_{1}=n_{2}=1$ is expected to be lowest in energy and gives rise to many-body states of $E^{\prime \prime}$ symmetry for both the parallel and antiparallel alignment [The remaining possibilities with two electrons in the same set of states are pushed up in energy by a larger Coloumb repulsion and have symmetries ${ }^{1} A_{1}^{\prime}+{ }^{3} A_{2}^{\prime}+{ }^{1} E^{\prime}$ for $n_{1}=2$ and ${ }^{1} A_{1}^{\prime}$ for

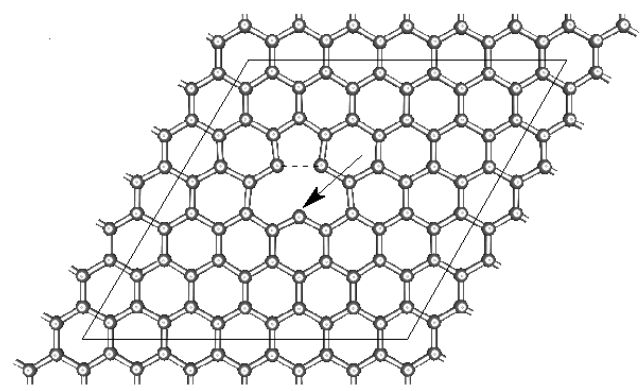

Figure 1: Optimized structure of a carbon vacancy in a $6 \times 6$ unit cell, with the apical carbon marked by an arrow.

$n_{2}=2$ ]. Thus, the ground-state is doubly degenerate for both spin alignments and undergoes (proper or pseudo) Jahn-Teller distortion. The latter occurs because of coupling with in-plane $e^{\prime}$ vibrations $\left(\left[E^{\prime \prime}\right]^{2}=\left[E^{\prime}\right]^{2}=A^{\prime}+E^{\prime}\right)$ which distort the symmetric arrangement of the carbon atoms around the vacancy. This is a standard $E \otimes e$ problem which is described by the so-called tricorn when such vibrations are included up to second-order ${ }^{31 / 32}$. Accordingly, a distorted geometry with a "pentagonal" ring and a "apical" carbon atom opposite to it (see Fig.1), can be predicted to be the equilibrium configuration (three-fold degenerate), as indeed found in several previous investigations $21+26$.

Out of plane, $E^{\prime \prime}$ vibrations do not lift degeneracy at first-order, but may affect energetics at higher orders, especially if coupling to the low-lying excited states is considered 32 . This is particularly important here since such distortions are qualitatively different for the states .. $\pi^{1} \sigma^{1}$ depending on whether the spins are parallel or antiparallel to each other. This is evident for the out-of-plane movement of the apical carbon atom in the distorted configuration: the $\sigma$ and the $\pi$ states may hybridize to some extent (and gain energy from double filling) if the two electrons couple at low-spin, otherwise they require extra energy to reduce their overlap. As a consequence, the planar structure is expected to be stable in the triplet state only; in the singlet, a non-planar configuration with the apical carbon atom slightly above (or below) the surface plane appears to be more stable. Because of that, the relative stability of the two spin states is geometry-dependent and its analysis requires at least investigating the outof-plane movement of the "apical" carbon atom. This is described in Section IV, after Section III has introduced the electronic structures methods and setups adopted, along with the structural models chosen to investigate the vacancy.

\section{METHODS AND MODELS}

Electronic structure calculations were performed at different correlation levels for different structural models. Periodic arrangements of vacancies in large 


\begin{tabular}{|c|c|c|c|c|c|c|}
\hline$n$ & $\triangle E(\mathrm{meV})$ & $M_{p}\left(\mu_{B}\right)$ & $d_{C C}^{p}(\AA)$ & $M_{n p}\left(\mu_{B}\right)$ & $d_{C C}^{n}(\AA)$ & $h_{C}(\AA)$ \\
\hline \hline 4 & 27.0 & 1.642 & 2.212 & 0.411 & 2.232 & 0.25 \\
\hline 5 & 38.2 & 1.889 & 2.126 & 0.111 & 2.169 & 0.28 \\
\hline 6 & 36.3 & 1.556 & 2.007 & 0.444 & 2.075 & 0.25 \\
\hline 7 & 30.2 & 1.556 & 1.999 & 0.444 & 2.026 & 0.24 \\
\hline 8 & 28.3 & 1.556 & 1.985 & 0.450 & 1.958 & 0.23 \\
\hline 9 & 26.9 & 1.556 & 1.978 & 0.446 & 1.969 & 0.23 \\
\hline 10 & 27.9 & 1.556 & 1.962 & 0.463 & 1.952 & 0.25 \\
\hline
\end{tabular}
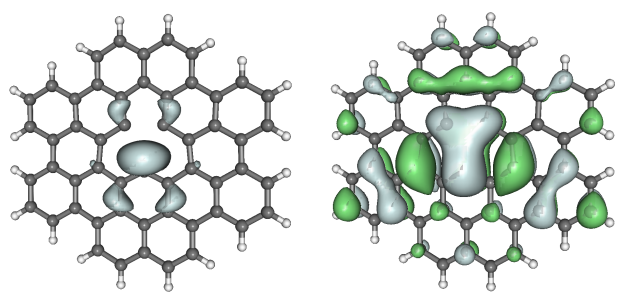

Table I: Results of full structural relaxation without constraints on the magnetization, for a vacancy in several $n \times n$ supercells. $\Delta E$ is the energy separation between the metastable non-planar $\left(C_{s}\right)$ structure and the planar $\left(C_{2 v}\right)$ minimum, $M_{n p}$ is the magnetization of the former and $M_{p}$ that of the latter. Also reported the length of newly formed $C C$ bond closing the pentagon $\left(d_{C C}^{p}\right.$ and $d_{C C}^{n}$ for planar and non-planar geometries, respectively) and the height $h_{C}$ of the apical carbon atom in the nonplanar configuration.

unit cells were investigated with standard, plane-wave based density functional theory calculations, whereas a finite-size (cluster) model was judiciously selected and studied with correlated wavefunction methods described below.

\section{A. Periodic models}

Periodic models were studied with plane-wave DFT as implemented in the Vienna ab initio package suite (VASP) 33/34. The exchange-correlation effects were treated with the Perdew-Burke-Ernzerhof (PBE) functional within the generalized gradient approximation (GGA), in the spin-polarized framework. KohnSham orbitals were expended on a plane-wave set limited to a $500 \mathrm{eV}$ energy cutoff and core electrons were frozen and replaced by projector-augmented wave (PAW) pseudopotentials $37 / 38$. Several $n \times n$ graphene supercells with a $20 \AA$ vacuum were initially considered to model the defective system, from $n=2$ to $n=10$, by using $\Gamma$ centered $k$-point meshes ranging from $15 \times 15 \times 1$ (for $n=2$ ) to $3 \times 3 \times 1$ for $n=6-10$. The structure of a vacancy in such cells was fully optimized without constraints on the magnetization and gave a Jahn-Teller distorted planar minimum, with a $C-C$ bond length in the pentagon of about $2 \AA$, i.e. smaller than the graphene lattice constant $a=2.46 \AA$ but much larger than a typical (single) $C-C$ bond $(1.54 \AA)$. Additionally, starting with a low-magnetization guess, we invariably found for $n \geq 4$ a metastable spin-polarized solution with vanishing magnetization and a non-planar configuration. Its energy separation $\Delta E$ from the planar minimum in the same supercell, along with the resulting magnetizations $M$ and the main geometrical parameters of the two structures are given in Table I] Results

Figure 2: The molecular model adopted for the wavefunction calculations, along with isosurfaces of singly occupied $\sigma$ (left) and $\pi$-midgap (right) orbitals for $|\phi|=0.015$ $\AA^{-3 / 2}$, as obtained at the restricted open-shell HartreeFock level $(S=1)$ for the minimum structure.

for the minimum are in good agreement with those found in previous studies $21+26$.

On the basis of these results we concluded that a $6 \times 6$ supercell with a $6 \times 6 \times 1 k$-point mesh was a good compromise between the need of reducing interaction between periodic images and computational manageability. Therefore, further investigations were performed with this setup.

\section{B. A finite-size model}

The semi-localized character of the electronic states induced by the vacancy makes it realistic the study of finite-size models where accurate, correlated wavefunction calculations are feasible. The size (and shape) of the cluster has to be large enough to minimize the effects that edges have on the details of the electronic structure, and small enough that complex many-body wavefunctions are yet tractable. To this end we considered a reasonably sized Polycyclic Aromatic Hydrocarbon $(\mathrm{PAH})\left(\mathrm{C}_{53} \mathrm{H}_{20}\right)$, a carbon cluster with a central vacant site which is hydrogenterminated at the edges (Fig. 2). Its actual shape was chosen -following the line of reasoning of Ref. 39. with the help of Tight-Binding (TB) calculations in such a way to limit the edge localization which does interfere with the defect-induced states at the Fermi level. In the chosen structure, edge states were found sufficiently far in energy from the vacancy-induced states (both at the TB and at the Hartree-Fock (HF) level) to make us confident that the resulting energetics accurately describes the vacancy. Cluster geometries were selected with a 'cut-out process' starting from the above mentioned $6 \times 6$ supercells, and adding hydrogen atoms to the undercoordinated edge $\mathrm{C}$ atoms, without further geometry refinement. In this way, comparison between the periodic and the cluster model with the same local arrangement close to the vacancy was possible.

Accurate results on the finite model were achieved through all-electron, correlated wavefunction calculations based on atom-centered basis-sets of the correla- 
tion consistent type (cc-pVDZ). Energy was obtained with the help of the MOLPRO suite of codes $\$ 40$ by correcting to second order in perturbation theory a 'reference' wavefunction of the Complete Active Space SelfConsistent Field (CASSCF) type, according to what is known as $\operatorname{CASPT} 2$ 41142. The $\operatorname{CASSCF}(n, m)$ wavefunction is a multi-determinant wavefunction containing all possible excitations of $n$ 'active' electrons in $m$ 'active' orbitals, where all the orbitals and expansion coefficients are variationally optimized $43 / 44$. For our purposes, we started with a minimal active space containing the $\sigma$ and $\pi$ orbitals localized on the vacancy and the two electrons occupying them at the HF level, and enlarged it by including two further $\pi$ orbitals (one below and one above the Fermi level), i.e. CAS $(4,4)$. We fully optimized the active orbitals and the thirty doubly-occupied orbitals higher in energy, and kept the lowest-lying (doubly occupied) orbitals frozen at the Hartree-Fock level. With the optimized CASSCF wavefunctions at hand, dynamic correlation was introduced by including perturbatively the effect of single and double excitations out of the configurations contained in the selected CAS 'reference' space.

\section{RESULTS}

\section{A. Periodic calculations}

As shown in TabI all the optimized structures show an appreciable magnetization, in agreement with previous results obtained for similar systems 21 , and this is significantly different for the two structures. The two geometries thus refer to the different electronic states -the high and the low spin state, respectively- which originate from the different ways the unpaired electrons localized on the vacancy align. Unfortunately, even assuming that the KS wavefunction correctly describes the spin state of the system, proper assignment is not possible with DFT. This is because (i) allowance of fractional occupation of single-particle Kohn-Sham states, as occurs with ensemble-DFT, typically prevents the appearance of pure states even when they are in principle possible at $T=0 \mathrm{~K}$, and (ii) pure but spin-polarized (unrestricted) solutions do not have a definite spin value $\left(S^{2}\right)$.

In order to get rid of these problems, and to mimic as much as possible the expected electron configurations, we relied on magnetization-constrained DFT calculations on the $6 \times 6$ supercell, setting the (projection of the) magnetic moment to two (zero) Bohr magnetons for the triplet (singlet) case. Full structural optimizations were then performed for different out-of-plane displacements $h$ of the apical carbon, for each 'spin' state, to investigate how these states evolve out of the plane.

The results of such calculations are shown in Fig.

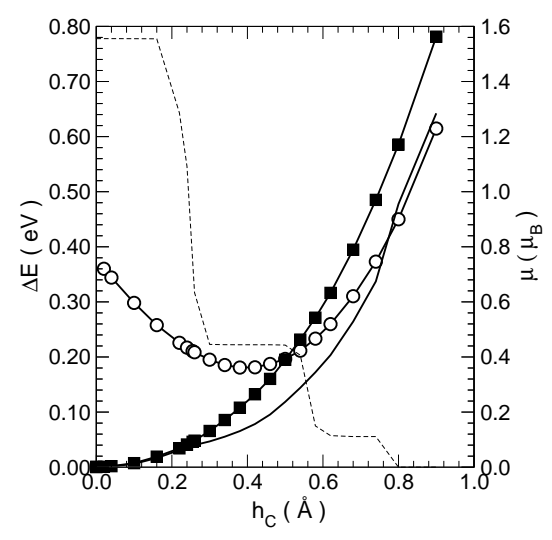

Figure 3: Magnetization-constrained energies as functions of the height $h$ of the apical carbon atom above the surface. Filled and empty symbols for $M=2,0 \mu_{B}$, referenced to the minimum of the $M=2 \mu_{B}$ case. Also reported as a thick line the results of magnetization-unconstrained calculations, referenced to their minimum, and the corresponding magnetization (dashed line, right scale).

3. referenced to the planar configuration in the triplet state, along with the magnetization-unconstrained curve referenced to its minimum (which is only 29 meV below that of the constrained triplet curve). It is clear that the latter is a mixture of the two electronic states, with the triplet prevailing for $h \approx 0$ and the singlet dominant for $h \gg 0$.

From Fig. 3, we also see that the global minimum belongs to the triplet curve and has a flat geometry (of symmetry $C_{2 v}$ ). The singlet curve instead shows two equivalent minima (of symmetry $C_{s}$ ) for the carbon atom above and below the plane, respectively, $\sim 0.4 \AA$ away from the surface. The latter thus represents a bi-stable system that crosses the triplet when the carbon atom moves out by about $\sim 0.5 \AA$, but is otherwise higher in energy. The energy difference between the singlet and triplet minima is $\sim 0.18 \mathrm{eV}$, thus significantly larger than the (unconstrained) $\triangle E$ for the same $6 \times 6$ supercell reported in TableI, which referred to 'mixed' electronic states. The singlet minima are separated by a barrier $\sim 0.2 \mathrm{eV}$ high which lies $\sim 0.4 \mathrm{eV}$ above the triplet. This estimate will be refined in the next section on the basis of more accurate electronic structure calculations. Before leaving this section we only stress that the curves reported in Fig. 3 refer to a full structural relaxation (in the given electronic state) with respect to all the degrees of freedom but the height of the apical carbon atom, and thus different geometries for the triplet and for the singlet typically result for the same $h$ value. The differences though are minimal as the height of the apical carbon atom is the main geometrical parameter controlling spin alignment in this system, hence graphs such as those of Fig. 3 are also representative of vertical energy differences. For instance, the pentagon CC bond length is $2.035 \AA$ in the triplet equi- 


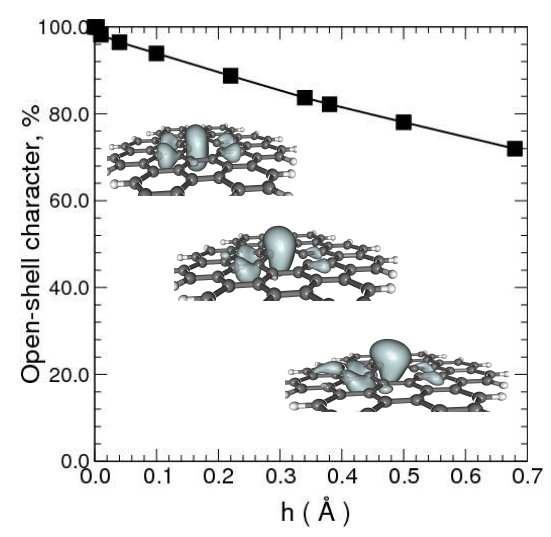

Figure 4: Variation of the open-shell character of the singlet state as the apical carbon atom moves out of plane. The symbols give the weight $\left(c_{1}^{2}+c_{2}^{2}\right) \times 100$ of the coefficients in the minimal $\mathrm{CAS}(2,2)$ wavefunction with diabatized orbitals described in the main text. The insets show isosurfaces at $|\phi|=0.015 \AA^{-3 / 2}$ of the corresponding $\sigma$-like CASSCF orbitals for representative values of $h=0.0,0.32,0.68 \AA$, from left to right, respectively.

librium configuration and increases to $2.081 \AA$ in the singlet minima, to be compared with $d_{C C}=2.007 \AA$ for the magnetization-free planar structure (Table I) and $d_{C C}=2.467 \AA$ in pristine graphene.

\section{B. Wavefunction calculations}

To analyze in detail the electronic structure in the neighborhoods of the vacancy we performed additional wavefunction calculations, as described in Section [II], on the geometries obtained at the DFT level, fit to the cluster model of Fig. 2. The use of a cluster introduces a number of issues related to the finiteness of the model (e.g. dependence on the system size, discretization of the continuum of states, etc. ) but removes some oddities of the supercell approach, e.g. the periodic repetition of defects in the same sublattice which would favor ferromagnetic alignment. We first checked that the singlet had the correct openshell character at the planar geometry, namely that the wavefunction reads approximately as

$$
\Psi_{h=0}^{S} \propto\left|\ldots \phi_{\sigma}^{\alpha} \phi_{\pi}^{\beta}\right| \pm\left|\ldots \phi_{\sigma}^{\beta} \phi_{\pi}^{\alpha}\right|
$$

where $\phi_{\sigma}$ and $\phi_{\pi}$ are $\sigma$-like and $\pi$-like orbitals on the apical carbon atoms, respectively, |..| is a shorthand for a Slater determinant and the plus (minus) sign holds for the triplet (singlet) state. For non-planar geometries $\sigma$-like and $\pi$-like orbitals get generally mixed, and the singlet displays both open- and closedshell character. Only if the first dominates the singlet can be considered to be the "same" electronic state of the triplet but with one spin flipped, and the singlettriplet energy separation is meaningful of an exchange coupling.

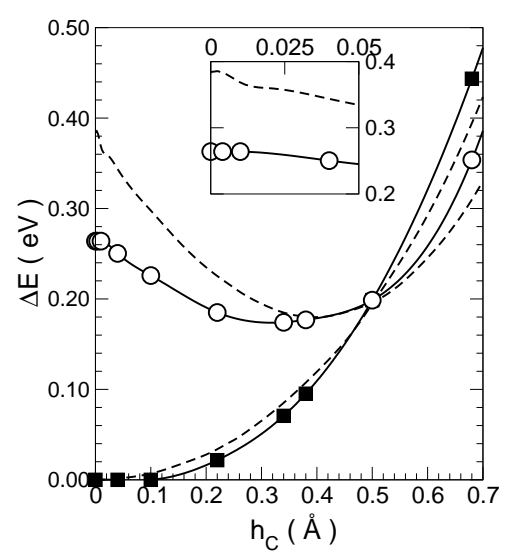

Figure 5: CASPT2 energies as function of the displacement $(h)$ of the apical carbon atom out of the surface plane, for the singlet (open symbols) and the triplet (filled symbols) states. Also reported for comparison the magnetization constrained DFT results of Fig 3 (dashed lines). Energies are referenced to the triplet minimum at the corresponding theory level. The inset shows a blow-up of the $h \approx 0$ region for the singlet.

To check this, we exploited the invariance of the CASSCF wavefunction with respect to rotations of the active orbitals, and choose orbitals which maximize the overlap (while keeping orthogonality) with the above $\phi_{\sigma}$ and $\phi_{\pi}$ states of the planar case. In this case

$\Psi_{h}^{S=0} \approx c_{1}\left|\ldots \phi_{\sigma}^{\alpha} \phi_{\pi}^{\beta}\right|+c_{2}\left|\ldots \phi_{\sigma}^{\beta} \phi_{\pi}^{\alpha}\right|+c_{3}\left|\ldots \phi_{\sigma}^{\alpha} \phi_{\sigma}^{\beta}\right|+c_{4}\left|\ldots \phi_{\pi}^{\alpha} \phi_{\pi}^{\beta}\right|$

where $c_{1}$ and $c_{2}=-c_{1}$ represent the open-shell contribution, and $c_{3}$ and $c_{4}$ account for the closed-shell character $\left(c_{3}=c_{4}=0\right.$ and $c_{2}=c_{1}$ in the triplet). In Fig. 4 we report the evolution of the weight $\left|c_{1}\right|^{2}+\left|c_{2}\right|^{2}$ in the normalized wavefunction, as a measure of the open-shell character in singlet state. They were obtained from simple $\operatorname{CAS}(2,2)$ calculations on the singlet-optimized geometries -i.e. using just the four-determinant wavefunction described above- but similar results are found for more elaborate functions. Evidently, the system is of open-shell type for a wide range of $h$ values, comprising the equilibrium one. Only for very large values of $h$ the system prefers a closed-shell configuration with "magnetic" properties turned off; correspondingly the triplet is pushed much higher in energy.

Multi-configuration SCF wavefunctions obtained distributing 4-electrons in 4-orbitals $(\mathrm{CAS}(4,4))$ were then optimized for several geometries sampled from the singlet and the triplet curves in Fig. 3, and used as references for perturbative (CASPT2) calculations. The results are shown in Fig. 5, together with DFT ones for comparison, for several values of the $h$ coordinate, referenced to the triplet minimum.

It is evident from Fig. 5 that CASPT2 and DFT results closely parallel each other for the triplet but differ substantially in the singlet. In the latter case, 
a cusp (due to a likely interaction with higher lying electronic states) is only present at the DFT level, and smooth out at the CASPT2 level, thereby signalling the presence of an avoided crossing. In fact, the CAS $(4,4)$ space is sufficiently large to allow us to properly describe a number of quasi-degenerate states, i.e. those obtained by placing all the four unpaired electrons of the vacancy in low-lying states.

As expected, ferromagnetic coupling is preferred for most values of the height of the carbon above the surface, and a crossing results at about $h=0.5 \AA$; for larger values of $h$, the gain in hybridization energy overcomes Coulomb repulsion, and the system show increased close-shell character. A minimum occurs in the singlet at $h=h_{0}=0.38 \AA\left(h_{0} \approx 0.4 \AA\right.$ at the DFT level), and is actually a double minimum, $h= \pm h_{0}$, on account of the meaning of the $h$ coordinate. The singlet is thus a symmetric bistable system with a barrier height of $E_{b}=0.09 \mathrm{eV}$.

Exchange (Hund) coupling was obtained by the vertical singlet-triplet energy separation, $J_{H}=\Delta E_{S T}$, using the geometries optimized for the triplet, and is shown in Fig 6 as a function of the angle $\theta$ subtended by the $\sigma$ dangling bond and the graphene plane. The results closely parallel those reported in Fig. 5 since, as observed above, the difference between singlet and triplet geometries are minimal. The coupling ranges from $J_{H} \sim 0.25 \mathrm{eV}$ in the planar configuration to about $J_{H} \sim 0.1 \mathrm{eV}$ in the equilibrium configuration of the singlet, and thus $J_{H} \sim 0.25-0.20 \mathrm{eV}$ seems to be appropriate for the ground-state system.

It is worth stressing at this point, that $J_{H}$ defined in this way is the Hund coupling constant related to the geometry-dependent $\sigma$-like and $\pi$-like orbitals hosting the unpaired electrons. Its value in the planar structure, $J_{H}^{0}=J_{H}(\theta \equiv 0)$, gives the Hund coupling constant in the Anderson impurity model for the vacancy $\sqrt{29}$, while its dependence on $\theta$ (at small angles) simply reflects the behavior of the hybridization strength ${ }^{45} V_{\sigma \pi}=\sqrt{2} t g(\theta) \sqrt{\left(1-2 t g^{2}(\theta)\right) / 3} \Delta \epsilon_{s p}$ ( $\Delta \epsilon_{s p}$ being the carbon $s-p$ splitting), as confirmed by the dashed line in Fig 6 .

Furthermore, despite the limited size (and discreteness of the energy spectrum) we found no indication that the $\pi$-midgap state is only marginally occupied, thereby suggesting that the limit $U_{\sigma \pi} \ll J_{H} / 4$ applies in the above mentioned Anderson model $\left(U_{\sigma \pi}\right.$ is the Coloumb repulsion between electrons in the $\sigma$ and in the $\pi$ midgap state). However, there exists the possibility that increasing the cluster size the situation reverts and the $\pi$-midgap state depopulates in favour of some other low-lying $\pi$-state ( $J=1 / 2$ problem).

\section{DISCUSSION}

Computed exchange coupling constants are clearly too large to have a decoupled response from the two

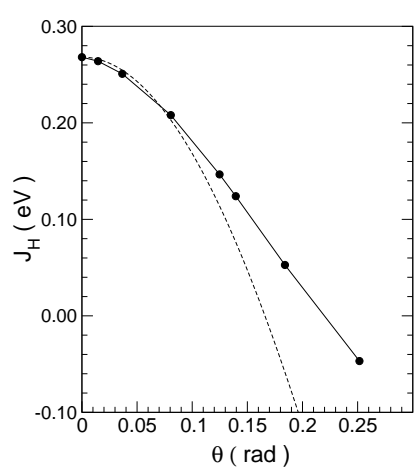

Figure 6: Variation of the Hund coupling constant as a function of the angle subtended by the $\sigma$ dangling bond and the graphene plane (filled symbols). Dashed line is a low-angle weighted-fit of the data to $J_{H}=J_{H}^{0}-$ $4 A t g^{2}(\theta)\left(1-2 t g^{2}(\theta)\right) / 3$, which gives $J_{H}^{0}=0.268 \mathrm{eV}$ and $A=7.59 \mathrm{eV}$.

localized electrons to external magnetic fields. The presence of a low-lying singlet at energy $\Delta$ above a $J$-paramagnetic ground-state does affect the magnetization, but only to the extent it modifies thermal populations, that is introducing a temperature- and fielddependent correction factor $f(\beta, H)=A\left(B e^{-\beta \Delta}+\right.$ $A)^{-1}$ to the thermally averaged magnetic moment (here $A=\sinh \left[\beta \gamma H\left(J+\frac{1}{2}\right)\right]$ and $B=\sinh \left[\frac{\beta \gamma H}{2}\right]$, $\beta=1 / k_{B} T$ as usual, $H$ is the magnetic field and $\gamma$ is the relevant gyromagnetic ratio). This factor has a distinguishing feature of making the moment no longer dependent on the reduced field $\beta H=H / k_{B} T$ only, but is hardly appreciable for $\beta \Delta \gtrsim 1$ (i.e. $T \lesssim 2000 \mathrm{~K}(!)$ for $\Delta \sim 0.2 \mathrm{eV})$. Only for $\beta \Delta \ll 1$ this factor transforms the $J=1$ ground-state magnetization density into twice that of a $J=1 / 2$ moment. In practice, this limit attains only if $\Delta$ is vanishing small, since the above (2-electron) "atomic" picture is challenged at much lower temperatures by thermal excitations out of/into the $\pi$ midgap state 50 . Likewise for doping which can be used to tune the $\pi$ state population, as it has been recently shown by molecular adsorption 27 .

All this suggests that the bare vacancy in freestanding graphene at low temperature should display a $J=1$ paramagnetic response and results reported by Ref. 8 are consistent with this picture. Decoupled $\sigma$ and $\pi$ moments, as those observed under bettercontrolled conditions by Nair et al! ${ }^{[27}$, though, are still plausible since the apical carbon atom may be easily stabilized out of the surface plane (at about $h \sim 0.3 \AA$ where $J_{H} \approx 0$ ), for instance in the presence of a (weakly-binding) substrate ${ }^{28}$ or ripples.

With the same token, spin-half residual moments may arise because of interaction with foreign species. Vacancies are highly reactive species which easily saturate their dangling $\sigma$ bond and leave a $\pi$ magnetic moment only. We have checked this by considering adsorption of a single hydrogen atom, and found that 
such a process is both thermodynamically and kinetically favored at any temperature: no barrier is indeed found in the adsorption profile and the binding energy is about twice that found for adsorption on a bulk site. A deeper analysis of single and multiple hydrogenation comprising static and dynamical aspects will be presented shortly.

Screening of the magnetic impurity by $\pi$-band states (Kondo effect) is a more subtle issue. DFT is not able to handle such highly correlated situations, and the finite model adopted for the wavefunction calculations, along with the limited excitations included in the wavefunction, prevent observation of any pairing between the "impurity" and the $\pi$ band states. In the finite size model, such pairing would be signalled by the presence of singly excited configurations where $\pi$ states singlet-couple with the impurity $\pi$-midgap or $\sigma$ state. Our wavefunction does include a number of excitations out of the occupied states in the CAS space and, perturbatively, excitations of the "core" states at the CASPT2 level, but a detailed analysis of the wavefunction such that presented above at the CASSCF level is out of question. In the overall triplet state, Kondo singlet-pairing would be signalled by an increasing delocalization of the spin-density when enlarging the cluster size but computational cost becomes prohibitive to check this [for the singlet the spin-density vanishes in any case on account of the vectorial character of this quantity]. Notice though that dynamical mean-field theory with local interactions showed no evidence of quenching of the $\pi$-related local magnetic moment $\frac{18}{18}$, in accordance with observations of spin- $\frac{1}{2}$ paramagnetism in fluorinated graphene ${ }^{7}$. In the case of vacancies, screening of the $\sigma$ moment is expected only for non planar geometries and, if any, is not compatible with Curielaw behavior observed in Ref.s 7/27. Metallic Kondo screening of spin- $\frac{1}{2}$ impurities has been used to explain transport measurements in irradiated graphene at different doping levels 10 , including charge neutrality, but the interpretation has been questioned $46 \mid 47$, and the observed logarithmic increase of resistivity at low temperatures related instead to electron-electron interactions in the disordered system $48 \mid 49$.

\section{CONCLUSIONS}

We reported on a detailed analysis of the electronic and geometric changes that occur upon vacancy formation in graphene, using both DFT and a high-level quantum chemistry method (CASPT2) to overcome known limitations of DFT. The picture that emerges is that two local magnetic moments coupled to each other to give a triplet ground-state, in accordance with a report of spin-1 paramagnetic species 8 . Spinhalf paramagnetism $\frac{727}{}$, though, can arise in many instances. Vacancies are highly reactive and easily saturate their $\sigma$ dangling bond in the presence of foreign species. Also, ripples or (weak) coupling to a substrate may stabilize a non-planar configuration of the apical carbon atom, thereby reducing the effective Hund coupling constant of the two-electron system and decouple the corresponding local moments. This is the likely source of spin-half paramagnetic behavior observed in Ref.s 7/27, where doping has been shown to effectively halve the magnetization density.

We could not deal with the possible pairing of the magnetic moment with the "conduction" $\pi$-band states, because of the limitation of DFT on one hand and the use of a finite cluster model (and limited excitation in the wavefunction) on the other hand. At the above level of theory we do not have indication, however, of such a coupling. This is consistent with the absence of anomalies in the measured susceptibility of Ref.s 727 , also at finite densities, and in that computed (for $\pi$-moments only) with dynamical mean field theory in the presence of local interactions ${ }^{18}$, and suggests that further investigation on the transport data measured by Ref. 10 is required for the Kondo effect in graphene to be unambiguously identified.
* Electronic address: rocco.martinazzo@unimi.it

1 M. Katsnelson, Graphene: Carbon in Two Dimensions (Cambridge University Press, 2012).

2 P. Esquinazi, A. Setzer, R. Höhne, C. Semmelhack, Y. Kopelevich, D. Spemann, T. Butz, B. Kohlstrunk, and M. Lösche, Phys. Rev. B 66, 024429 (2002).

3 P. Esquinazi, D. Spemann, R. Höhne, A. Setzer, K.-H. Han, and T. Butz, Phys. Rev. Lett. 91, 227201 (2003).

4 J. Barzola-Quiquia, P. Esquinazi, M. Rothermel, D. Spemann, T. Butz, and N. Garcia, Phys. Rev. B 76, 161403 (2007).

5 Y. Wang, Y. Huang, Y. Song, X. Zhang, Y. Ma, J. Liang, and Y. Chen, Nano Lett. 9, 220 (2009).

${ }^{6}$ M. Sepioni, R. R. Nair, S. Rablen, J. Narayanan, F. Tuna, R. Winpenny, A. K. Geim, and I. V. Grig- orieva, Phys. Rev. Lett. 105, 207205 (2010).

7 R. R. Nair, M. Sepioni, I.-L. Tsai, O. Lehtinen, J. Keinonen, A. V. Krasheninnikov, T. Thomson, A. K. Geim, and I. V. Grigorieva, Nature Phys. 8, 199 (2012).

8 A. Ney, P. Papakonstantinou, A. Kumar, N.-G. Shang, and N. Peng, Appl. Phys. Lett. 99, 102504 (2011).

${ }^{9}$ K. M. McCreary, A. G. Swartz, W. Han, J. Fabian, and R. K. Kawakami, Phys. Rev. Lett. 109, 186604 (2012).

10 J.-H. Chen, L. Li, W. G. Cullen, E. D. Williams, and M. S. Fuhrer, Nature Phys. 7, 535 (2011).

11 M. Inui, S. A. Trugman, and E. Abrahams, Phys. Rev. B 49, 3190 (1994).

12 S. Fajtlowicz, P. E. John, and H. Sachs, Croat. Chem. Acta 78, 195 (2005).

13 V. M. Pereira, F. Guinea, J. Lopes dos Santos, N. Peres, 
and A. Castro Neto, Phys. Rev. Lett. 96, 036801 (2006).

14 V. M. Pereira, J. M. B. Lopes dos Santos, and A. H. Castro Neto, Phys. Rev. B 77, 115109 (2008).

15 M. M. Ugeda, I. Brihuega, F. Guinea, and J. M. GomezRodriguez, Phys. Rev. Lett. 104, 096804 (2010).

16 E. H. Lieb, Phys. Rev. Lett. 62, 1201 (1989).

17 E. Kogan, Phys. Rev. B 84, 115119 (2011).

${ }^{18}$ P. Haase, S. Fuchs, T. Pruschke, H. Ochoa, and F. Guinea, Phys. Rev. B 83, 241408(R) (2011).

19 L. Hornekær, E. Rauls, W. Xu, Z. Šljivančanin, R. Otero, I. Stensgaard, E. Læegsgaard, B. Hammer, and F. Besenbacher, Phys. Rev. Lett. 97, 186102 (2006).

20 S. Casolo, O. M. Løvvik, R. Martinazzo, and G. F. Tantardini, J. Chem. Phys. 130, 054704 (2009).

21 A. A. El-Barbary, R. H. Telling, C. P. Ewels, M. I. Heggie, and P. R. Briddon, Phys. Rev. B 68, 144107 (2003).

22 P. O. Lehtinen, A. S. Foster, Y. Ma, A. V. Krasheninnikov, and R. M. Nieminen, Phys. Rev. Lett. 93, 187202 (2004).

23 O. V. Yazyev and L. Helm, Phys. Rev. B 75, 125408 (2007).

${ }^{24}$ M. Dharma-wardana and M. Z. Zgierski, Physica E: Low-dimensional Systems and Nanostructures 41, 80 (2008).

25 X. Q. Dai, J. H. Zhao, M. H. Xie, Y. N. Tang, Y. H. Li, and B. Zhao, Eur. Phys. J. B 80, 343 (2011).

26 J. J. Palacios and F. Yndurain, Phys. Rev. B 85, 245443 (2012).

27 R. R. Nair, I.-L. Tsai, M. Sepioni, O. Lehtinen, J. Keinonen, A. V. Krasheninnikov, A. H. C. Neto, A. K. Geim, and I. V. Grigorieva, arXiv:1301.7611 (2013).

28 M. M. Ugeda, D. Fernández-Torre, I. Brihuega, P. Pou, A. J. Martínez-Galera, R. Pérez, and J. M. GómezRodríguez, Phys. Rev. Lett. 107, 116803 (2011).

29 M. A. Cazalilla, A. Iucci, F. Guinea, and A. H. C. Neto, arXiv:1207.3135 (2012).

30 A. K. Mitchell and L. Fritz, arXiv:1212.2631 (2012).

31 I. B. Bersuker, Chem. Rev. 101, 1067 (2001).
${ }^{32}$ I. B. Bersuker, The Jahn-Teller effect (Cambridge University Press, 2006).

33 G. Kresse and J. Hafner, Phys. Rev. B 49, 14251 (1994).

${ }^{34}$ G. Kresse and J. Furthmüller, Phys. Rev. B 54, 11169 (1996).

35 J. P. Perdew, K. Burke, and M. Ernzerhof, Phys. Rev. Lett. 77, 3865 (1996).

36 J. P. Perdew, K. Burke, and M. Ernzerhof, Phys. Rev. Lett. 78, 1396 (1997).

37 P. E. Blöchl, Phys. Rev. B 50, 17953 (1994).

38 G. Kresse and D. Joubert, Phys. Rev. B 59, 1758 (1999).

39 M. Bonfanti, S. Casolo, G. F. Tantardin, A. Ponti, and R. Martinazzo, J. Chem. Phys. 135, 164701 (2011).

40 H.-J. Werner, P. J. Knowles, G. Knizia, F. R. Manby, M. Schütz, et al. (2010).

41 H. J. Werner, Mol. Phys. 89, 645 (1996).

42 P. Celani and H. J. Werner, J. Chem. Phys. 112, 5546 (2000).

43 H.-J. Werner and P. J. Knowles, J. Chem. Phys. 82, 5053 (1985).

44 P. J. Knowles and H.-J. Werner, Chem. Phys. Letters 115, 259 (1985).

${ }^{45}$ L. Pauling, The Nature of the Chemical Bond (Cornell University Press, Ithaca, N.Y., 1972).

46 J. Jobst and H. B. Weber, Nature Phys. 8, 352 (2012).

47 J.-H. Chen, L. Li, W. G. Cullen, E. D. Williams, and M. S. Fuhrer, Nature Phys. 8, 353 (2012).

48 B. L. Altshuler, A. G. Aronov, and P. A. Lee, Phys. Rev. Lett. 44, 1288 (1980).

49 J. Jobst, D. Waldmann, I. V. Gornyi, A. D. Mirlin, and H. B. Weber, Phys. Rev. Lett. 108, 106601 (2012).

50 Notice though that thermal excitations are not relevant to magnetometry experiments since they are performed at very low temperatures. This is confirmed by the fact that, under similar conditions, flourinated graphene gives the expected spin $-\frac{1}{2}$ response of $\pi$ moments ${ }^{7}$. Double occupation of the $\sigma$ state, on the other hand, is prevented by the large Coulomb repulsion $\frac{29]}{,} U_{\sigma \sigma} \approx 10$ $\mathrm{eV}$, and the binding energy in this state is substantial26, $\epsilon_{\sigma} \approx-0.75 \mathrm{eV}$. 